IFUM - 94 / 455/ January - FT

\title{
Passive scalar convection in 2D long-range delta-correlated velocity field: Exact results.
}

\author{
Michael Chertkov ${ }^{1}$, Yan V. Fyodorov ${ }^{1}{ }^{2}$, and Igor Kolokolov ${ }^{3} 4$ \\ ${ }^{1}$ Department of Physics of Complex Systems, Weizmann Institute of Science, Rehovot 76100, \\ Israel \\ 2 Petersburg Nuclear Physics Institute, 188350 Gatchina, St.Petersburg, Russia \\ ${ }^{3}$ Budker Institute for Nuclear Physics, Novosibirsk 630 090, Russia \\ ${ }^{4}$ INFN, Sezione di Milano, 20133 Milano, via Celoria 16, Italy
}

(March 17, 2018)

\begin{abstract}
The letter presents new field-theoretical approach to 2D passive scalar problem. The Gaussian form of the distribution for the Lyapunov exponent is derived and its parameters are found explicitly.
\end{abstract}

PACS numbers 47.10, 47.25C, 02.30.C

Typeset using REVTEX 


\section{DEFINITION OF THE MODEL}

The convection of a passive scalar (PS) in 2D by an external long-range velocity field is well-defined linear problem [1,2]. To solve the problem is to find all correlation functions of the passive scalar field $w(\mathbf{r} ; t)$ governed by the following equation

$$
\dot{\omega}+u_{\alpha} \nabla_{\alpha} \omega=\phi
$$

with $\vec{u}(\mathbf{r} ; t)$ being an external Eulerian long-range $\left(q<L^{-1}\right)$ velocity field and $\phi$ being a random external source localized in $k$ space at $k_{0}=L^{-1}$. The measure of averaging over the random source in the simplest case of white-noise source statistics can be chosen in the following form

$$
\begin{aligned}
& \mathcal{D} \phi \exp \left(-\frac{\int_{-\infty}^{+\infty} \phi_{k=1 / L}^{2}(t) d t}{2 P_{2}}\right) \quad, \quad \phi_{k}=(2 \pi)^{-2} \int \phi(\mathbf{r} ; t) \exp (i \mathbf{k r}) d \mathbf{r}, \\
& <\phi_{\mathbf{k}}(t) \phi_{\mathbf{k}^{\prime}}\left(t^{\prime}\right)>_{s} \equiv \Xi_{\mathbf{k}-\mathbf{k}^{\prime}}\left(t-t^{\prime}\right)=\frac{P_{2}}{2 \pi k_{0}} \delta\left(t-t^{\prime}\right) \delta\left(\mathbf{k}-\mathbf{k}^{\prime}\right) \delta\left(k-k_{0}\right),
\end{aligned}
$$

without any loss of generality as it was shown in [3]. Here $P_{2}$ stands for the flow of the squared PS on the source.

In order to eliminate a homogeneous sweeping it is convenient to pass to the locally comoving frame [四] expressing the Eq. (四) in terms of the Quasi-Lagrangian (QL) velocities related to the initial Eulerian ones by means of the formula

$$
\mathbf{u}(\mathbf{r} ; t)=\mathbf{v}\left(\mathbf{r}-\int^{t} \mathbf{v}(0 ; \tau) d \tau ; t\right)
$$

The eq.(1) takes correspondingly the form

$$
\dot{\omega}+\left(v^{\alpha}-v_{0}^{\alpha}\right) \nabla_{\alpha} \omega=\phi, \quad \mathbf{v}_{0}=\mathbf{v}(0 ; \tau)
$$

Following the ideas of recent paper [3] one can expand $v^{\alpha}(\mathbf{r})-v^{\alpha}(0)=\sigma^{\alpha \beta} r^{\beta}$ for the points satisfying $r<L$. Here $\hat{\sigma}$ is the random in time traceless [5] symmetric [6] matrix. For the white-noise velocity field statistics (which is the only case considered in the present paper) the $\hat{\sigma}$ elements measure has the form providing space-isotropy of the simultaneous pair correlator of the $\mathrm{QL}$ velocities 


$$
\mathcal{D} \hat{\sigma}(t)=\mathcal{D}\left(\begin{array}{cc}
a & b \\
b & -a
\end{array}\right)=\mathcal{D} a \mathcal{D} b \exp \left(-S_{0}\right), S_{0}=\frac{1}{2 D} \int\left(a^{2}+b^{2}\right) d t
$$

To solve the resulting equation

$$
\dot{\omega}+\sigma^{\alpha \beta} r^{\beta} \nabla_{\alpha} \omega=\phi
$$

one performs the substitution $\omega(\mathbf{r} ; t)=\psi(\mathbf{R} ; t), R^{\alpha}=W^{\alpha \beta}(t) r^{\beta}$ that allows to write down the solution of the eq. (6) in the following form

$$
\omega(\vec{r} ; t)=\psi(\mathbf{R}(t) ; t)=\int_{-\infty}^{t} d \tau \phi\left(\hat{W}^{-1}(\tau,-\infty) \mathbf{R}(t) ; \tau\right) \equiv \int_{-\infty}^{t} d \tau \phi(\hat{W}(t, \tau) \mathbf{r} ; \tau)
$$

where the matrix $\hat{W}(t ; \tau)$ can be sought for as satisfying the relation

$$
\dot{\hat{W}}_{t}+\hat{W} \hat{\sigma}=0
$$

with initial condition $\hat{W}(\tau, \tau)=\hat{1}$. Due to Eq.(可) the all order correlation functions of the PS field may be rewritten in terms of the correlation functions of the source field $\phi$. For example, the pair correlation function is

$$
<\omega\left(\mathbf{r}_{1} ; t_{1}\right) \omega\left(\mathbf{r}_{2} ; t_{2}\right)>=\int_{-\infty}^{t_{1}} d \tau_{1} \int_{-\infty}^{t_{2}} d \tau_{2} \Xi\left(\hat{W}\left(t_{1}, \tau_{1}\right) \mathbf{r}_{1}-\hat{W}\left(t_{2}, \tau_{2}\right) \mathbf{r}_{2} ; \tau_{1}-\tau_{2}\right)_{\sigma}
$$

The explicit form of the simultaneous pair correlator ( which has to be isotropic) can be extracted from Eqs.(9) and (2) as

$$
<\omega\left(\mathbf{r}_{1} ; t\right) \omega\left(\mathbf{r}_{2} ; t\right)>=P_{2} \int_{0}^{\infty} d t J_{0}\left(\frac{\left|\hat{W}(t, 0)\left(\mathbf{r}_{1}-\mathbf{r}_{2}\right)\right|}{L}\right),
$$

with $J_{0}(x)$ being the Bessel function of zeroth order.

So, following the calculations of the paper [3], we reduced our initial problem to the evaluation of some functions of the matrix $\hat{W}$ satisfying the Eq.(8). The statistics of matrix elements of $\hat{W}(t, 0)$ is defined by the ensemble of random real traceless symmetric matrices $\hat{\sigma}$, see Eq.(5). The authors of the paper [3] have calculated $<|\hat{W}(t, 0) r|>$ by means of the direct expansion of the anti-chronological $T$ - exponent that is the formal solution of the Eq.(8). It gave them possibility to estimate the simultaneous pair correlator (10) at 
small distances $r_{1}-r_{2}$ as $\frac{P_{2}}{D} \ln \left(\frac{L}{r}\right)$. In addition, analyzing the set of many-point correlation functions of PS field Falkovich and Lebedev were able to demonstrate that the instantaneous statistics of such a field becomes more close to the gaussian one when passing down-scales.

In the present paper we suggest a new nonperturbative field-theoretical approach to the calculation of the PS-field correlations. We show that the asymptotic behavior of the pair correlator Eq. (10) is governed by the self-averaging (or "deterministic") quantity: the Lyapunov exponent. By means of our method we rederive, extend and exactly prove all abovementioned results by Falkovich and Lebedev. In particular, we find explicitly the value of the variance of the gaussian distribution characterizing the short range PS-field statistics.

\section{FUNCTIONAL REPRESENTATION FOR AVERAGED FUNCTIONALS OF $\hat{W}$}

The matrix $\hat{W}(t)$ can be extracted from Eq.(8) only in a form of the anti-chronological time-ordered exponent

$$
\hat{W}(t, 0)=\tilde{T} \exp \left(-\int_{0}^{t} d t^{\prime} \hat{\sigma}\left(t^{\prime}\right)\right), \hat{W}(0,0)=\hat{1}
$$

rather than in terms of some regular function of $\hat{\sigma}$. A similar problem - transformation of a time-ordered exponent of some linear combination of spin $S U(2)$ operators (arising when one tries to write down an exact functional representation for the partition function for Quantum Heisenberg ferromagnet) has been solved by Kolokolov [7]. The main idea of the method is to introduce such a new set of integration variables in the functional integral that $\tilde{T} \exp$ becomes some regular function when expressed in their terms. In the present context we use a new modification of the Ansatz proposed in [7] expanding the $\sigma$ matrix over the spin $2 \times 2$ matrices as

$$
\hat{\sigma}=a \hat{\sigma}_{z}+b \hat{\sigma}_{x} .
$$

First, we introduce a new basis of the spin algebra:

$$
\hat{\sigma}_{y}=\left(\begin{array}{cc}
0 & -i \\
i & 0
\end{array}\right), \hat{\sigma}_{+}=\hat{\sigma}_{z}+i \hat{\sigma}_{x}=\left(\begin{array}{cc}
1 & i \\
i & -1
\end{array}\right), \hat{\sigma}_{-}=\hat{\sigma}_{z}-i \hat{\sigma}_{x}=\left(\begin{array}{cc}
1 & -i \\
-i & -1
\end{array}\right),
$$


that corresponds to the rotation of the quantization axis from the usual orientation (parallel to the z-axis) to the new one parallel to the y-axis. Instead of the fields $a(t), b(t)$ we choose the new ones $\varphi^{ \pm}=(a \pm i b) / 2$ transforming $\hat{\sigma}$ and the integration measure Eq.(5) to the more compact form:

$$
\hat{\sigma}=\varphi^{-} \hat{\sigma}_{+}+\varphi^{+} \hat{\sigma}_{-} \quad ; \quad \mathcal{D} \hat{\sigma}(t)=\mathcal{D} \varphi^{ \pm} \exp \left(-S_{0}\right), S_{0}=\frac{2}{D} \int_{0}^{+\infty} \varphi^{+} \varphi^{-} d t
$$

Let us now introduce the operator given in the explicit form as

$$
\begin{aligned}
& \hat{A}(t, 0)=\exp \left[-\hat{\sigma}_{-} \psi^{+}(0)\right] \exp \left[-\hat{\sigma}_{+} \int_{0}^{t} \psi^{-}\left(t^{\prime}\right) e^{2 \int_{0}^{t^{\prime}} \rho\left(t^{\prime \prime}\right) d t^{\prime \prime}} d t^{\prime}\right] \exp \left[\hat{\sigma}_{y} \int_{0}^{t} \rho\left(t^{\prime}\right) d t^{\prime}\right] \times \\
& \exp \left[\hat{\sigma}_{-} \psi^{+}(t)\right]
\end{aligned}
$$

where $\psi^{ \pm}, \rho$ are some new dynamical fields. Using the commutations relations for the spin operators $\sigma_{ \pm}, \sigma_{y}$ it is easy to check that the operator $\hat{A}$ obeys the differential equation

$$
\dot{\hat{A}}_{t}=\hat{A}\left[-\hat{\sigma}_{+} \psi^{-}+\hat{\sigma}_{-}\left(4 \psi^{-}\left(\psi^{+}\right)^{2}+2 \rho \psi^{+}+\dot{\psi}^{+}\right)+\hat{\sigma}_{y}\left(-4 \psi^{-} \psi^{+}+\rho\right)\right]
$$

Using the fact that the first exponential factor in Eq.(15) makes the operator $\hat{A}$ satisfying the condition $\hat{A}(0,0)=1$ and comparing the last equation (16) with the equivalent one (8) we find out the change of variables

$$
\begin{aligned}
& \varphi^{-}=\psi^{-}, \\
& \varphi^{+}=-\dot{\psi^{+}}+4 \psi^{-}\left(\psi^{+}\right)^{2} ; \\
& \rho=4 \psi^{-} \psi^{+},
\end{aligned}
$$

in case of which dynamical matrices $\hat{W}$ and $\hat{A}$ coincide. It allows us to obtain the explicit functional integral representation for any average written in terms of $\hat{W}$ by changing variables from $\varphi^{ \pm}$to $\psi^{ \pm}$. The Jacobian of the map Eq.([17) is

$$
\mathcal{D} \varphi^{ \pm}=\mathcal{J}\left[\psi^{ \pm}\right] \mathcal{D} \psi^{ \pm}
$$

and it is essentially dependent on the kind of regularization and a type of conditions imposed on the field $\psi^{+}$. The matter is that the transformation Eq. (17) contains the derivative of the 
field $\psi^{+}$with respect to time in its right-hand side. Therefore, it should be supplied with some initial or boundary conditions. In the papers [7] it has been shown that only imposing conditions of the initial kind it is possible to ensure the invertability of the map Eq.(17). Another point is that in the course of calculation it is necessary to average some functions of the operator $A(T, 0)$ at fixed time moment $T$ over the measure Eq.(14). For a given $T$ it is convenient to fix the final value of the field $\psi^{+}$

$$
\psi^{+}(T)=-\frac{1}{2}
$$

Then the operator $A(T, 0)$ acting on the initial vector $\left(\begin{array}{l}1 \\ 0\end{array}\right)$ produces the following simple expressions:

$$
\begin{aligned}
& \vec{R}(T)=\hat{A}(T, 0)\left(\begin{array}{c}
1 \\
0
\end{array}\right)=\frac{1}{2} e^{4 \int_{0}^{T} \psi^{+} \psi^{-} d t}\left(\begin{array}{c}
1-2 \psi^{+}(0) \\
i\left(1+2 \psi^{+}(0)\right.
\end{array}\right), \\
& \vec{R}^{2}(T)=-2 \psi^{+}(0) e^{8 \int_{0}^{T} \psi^{+} \psi^{-} d t}
\end{aligned}
$$

Here we exploited the isotropy condition and substituted $\vec{R}(0)=\left(\begin{array}{l}1 \\ 0\end{array}\right)$ for the initial value of the vector $R(T)$ without any loss of generality.

The regularization of the map Eq.(17) (again as in the papers [7,8) is determined by the physical argumentation. Here it stems from the translation in time invariance of the white-noise measure Eq.(14). Indeed, the equality

$$
<\varphi^{+}(T) \int^{T} \varphi^{-}(t) d t>=<\varphi^{-}(T) \int^{T} \varphi^{+}(t) d t>
$$

leads to the extension of the definition of the step function $\theta(x)$ at $x=0$ such that $\theta(0)=1 / 2$. Thus, the discrete version of the map (14) $\left(\varphi_{n}^{ \pm}=\varphi^{ \pm}\left(t_{n}\right) ; n=1, \ldots, M ; \epsilon=\frac{T}{M} \rightarrow 0 ; t_{n}=\right.$ $\epsilon n ; M \rightarrow \infty)$, compatible with the symmetry condition Eq. 23 is

$$
\varphi_{n}^{-}=\psi_{n}^{-}, \varphi_{n}^{+}=-\frac{1}{\epsilon}\left(\psi_{n+1}^{+}-\psi_{n}^{+}\right)+\psi_{n}^{-}\left(\psi_{n}^{+}+\psi_{n+1}^{+}\right)^{2}
$$

that gives the following expression for the Jacobian 


$$
\mathcal{J}=\text { const } \exp \left(4 \int_{0}^{T} \psi^{+} \psi^{-} d t^{\prime}\right)
$$

When calculating the Jacobian Eq.(25) the fields $\varphi^{ \pm}$and $\psi^{ \pm}$were considered to be independent complex variables or, in other words, as different coordinate systems in the whole space $\mathcal{C}^{2 M}$ of the fields' configurations. The conditions

$$
\varphi^{+}=\left(\varphi^{-}\right)^{*}
$$

being externally imposed on the model specify the surface $\Sigma$ in $\mathcal{C}^{2 M}$ along which the differential forms $\mathcal{D} \varphi^{+} \wedge \mathcal{D} \varphi^{-}$or $\mathcal{D} \psi^{+} \wedge \mathcal{D} \psi^{-}$are integrated. For the coordinate sets $\left(\psi^{ \pm}\right)$the equation (17) for $\Sigma$ can be considered to be an implicit one. According to the Cauchy-Poincare theorem the integration surface can be deformed in an arbitrary way in the convergence domain provided we integrate an analytical function. There exists a continuous family of surfaces (homotopy) which includes both the surfaces $\Sigma$ and the "standard" one $\Sigma^{\prime}$ :

$$
\Sigma^{\prime}=\left\{-\psi^{+}=\left(\psi^{-}\right)^{*}\right\}
$$

The explicit expression for such a homotopy in a more general case can be found in the paper [9]. Thus, we can replace the surface of integration $\Sigma$ by the standard one $\Sigma^{\prime}$.

Substituting Eq.(17) into the measure Eq.(5) and using the expressions Eqs. (19,25) we obtain the following modification of the measure for averaging over $\psi^{ \pm}$[10]:

$$
N \mathcal{D} \psi^{ \pm} \exp \left(-S_{1}\left\{\psi^{ \pm}\right\}\right), S_{1}=\int_{0}^{+\infty}\left[-\frac{2}{D} \dot{\psi}^{+} \psi^{-}+\frac{8}{D}\left(\psi^{-} \psi^{+}\right)^{2}-4 \psi^{+} \psi^{-}\right] d t
$$

or , correspondingly, in the discrete form

$$
S_{1}=\sum_{n}\left[-\frac{2}{D}\left(\psi_{n+1}^{+}-\psi_{n}^{+}\right) \psi_{n}^{-}+\frac{2}{D} \epsilon\left(\psi_{n}^{-}\right)^{2}\left(\psi_{n}^{+}+\psi_{n+1}^{+}\right)^{2}-2 \epsilon \psi_{n}^{-}\left(\psi_{n}^{+}+\psi_{n+1}^{+}\right)\right]
$$

It means that we reformulated the initial problem of the multiplicative random matrix process with the measure Eq.(5) to the multiplicative random scalar process with the measure given by Eq.(28). 


\section{GAUSSINESS OF PASSIVE SCALAR CORRELATIONS}

Another peculiarity of an arbitrary multiplicative random process is the Gaussian-like fluctuations of the Lyapunov exponent $\lambda(T)$ with an amplitude that decays like const $/ \sqrt{T}$ when $T$ goes to infinity. Let us note that this fact has been proven both for the multiplicative

random scalar process (see, e.g. [11]) and for matrix one (see [12].) The usual way to calculate the constant in front of $1 / T^{1 / 2}$ is to restore the distribution function of $\lambda(t)$ at arbitrary time from the set of moments $\left\langle R^{2 n}\right\rangle$ and then to extract the Gaussian-like peak around the mean Lyapunov exponent at large enough time (see for example the calculation of such a kind in $1 D$ [13] and quasi $1 D$ [14 localization problem). We follow the same way in the present context as well.

The Lyapunov exponent in our case is convenient to define as

$$
\lambda \equiv \frac{\ln \left[\vec{R}^{2}(T)\right]}{2 T}
$$

In order to find the probability distribution function (PDF) of $\lambda$ let us firstly calculate all the even moments of $\vec{R}$

$$
R_{m}(T) \equiv<R^{2 m}>=\frac{<\left(-2 \psi^{+}(0)\right)^{m} e^{8 m \int_{0}^{T} \psi^{+} \psi^{-} d t}>_{1}}{<1>_{1}}
$$

where $\langle\ldots\rangle_{1}$ stands for the average with respect to the measure Eq. (28). Passing to the new integration variables by means of the gauge transformations

$$
\psi^{ \pm}=\chi^{ \pm} \exp \left[\mp 4 \int_{t}^{T} \chi^{+} \chi^{-} d t^{\prime} \pm(2 m+1) D(T-t)\right]
$$

that can be written in the discretized form as

$$
\begin{aligned}
& \psi_{n}^{+}=\chi_{n}^{+} \exp \left[-2 \sum_{j=n}^{M} \epsilon\left(\chi_{j+1}^{+}+\chi_{j}^{+}\right) \chi_{j}^{-}+(2 m+1) \epsilon D(M-n+1)\right] \\
& \psi_{n}^{-}=\chi_{n}^{-} \exp \left[2 \sum_{j=n+1}^{M} \epsilon\left(\chi_{j+1}^{+}+\chi_{j}^{+}\right) \chi_{j}^{-}+\epsilon\left(\chi_{n}^{+}+\chi_{n+1}^{+}\right) \chi_{n}^{-}-(2 m+1) \epsilon D(M-n)-\right. \\
& \left.\frac{1}{2} \epsilon(2 m+1) D\right]
\end{aligned}
$$


we reduce the averaging in both numerator and denominator (the latter case corresponds to $m=0)$ of Eq.(31) to the Gaussian-type ones

$$
<\left(-2 \psi^{+}(0)\right)^{m} e^{8 m \int_{0}^{T} \psi^{+} \psi^{-} d t}>_{1}=(-2)^{m}<\left(\chi^{+}(0)\right)^{m}>_{2} e^{\left[(2 m+2) m+\frac{1}{2}\right] D T},<1>_{1}=e^{\frac{D T}{2}}
$$

Here $\langle\ldots\rangle_{2}$ stands for the averaging with respect to the Gaussian measure

$$
N^{\prime} \mathcal{D} \chi^{ \pm} \exp \left(-S_{2}\left\{\chi^{ \pm}\right\}\right), S_{2}=\frac{2}{D} \int_{0}^{+\infty} \dot{\chi}^{+} \chi^{-} d t
$$

and one takes into account that the Jacobian of the map Eq.(32) is

$$
J[\chi]=\exp \left[-2 \int_{0}^{T} \chi^{+} \chi^{-}+\left(m+\frac{1}{2}\right) D(T-t)\right]
$$

The average $<\left(\chi^{+}(0)\right)^{m}>_{2}$ is equal to $\left(-\frac{1}{2}\right)^{m}$ due to condition $\chi^{+}(T)=-\frac{1}{2}$. This result is easy to get shifting $\chi^{+} \rightarrow-\frac{1}{2}+\tilde{\chi}^{+}$and noticing that all the averages of $\tilde{\chi}^{+}$are equal to zero. Thus, we arrive at the result

$$
R_{m}(T)=e^{D T(2 m+2) m}
$$

Knowing $R_{m}$ one can extract Fourier-representation for PDF of $R^{2}$ in the following way:

$$
\tilde{\mathcal{P}}_{k}=\sum_{m=0}^{\infty} \frac{(i k)^{m}}{m !} R_{m}=\int_{-\infty}^{+\infty} \frac{e^{-D T / 2}}{\sqrt{8 \pi D T}} \exp \left[-\frac{x^{2}}{8 D T}+\frac{x}{2}+i k e^{x}\right)
$$

and the PDF for $R^{2}$ is obtained after the Fourier inversion in the form

$$
\tilde{\mathcal{P}}\left(R^{2}\right)=\int_{-\infty}^{+\infty} \frac{d k}{2 \pi} e^{-i k z} \tilde{\mathcal{P}}_{k}=\int_{-\infty}^{+\infty} d x \frac{e^{-D T / 2}}{\sqrt{8 \pi D T}} \exp \left[-\frac{x^{2}}{8 D T}+\frac{x}{2}\right] \delta\left(R^{2}-e^{x}\right)
$$

Correspondingly, the PDF the Lyapunov exponent defined in Eq.(30) is explicitly given by the expression

$$
\mathcal{P}(\lambda)=\sqrt{\frac{T}{2 \pi D}} e^{-\frac{T(\lambda-D)^{2}}{2 D}}
$$

that means exact Gaussian fluctuations around the average value $<\lambda>=D$ with the variance $\frac{D}{T}$ vanishing when $T$ tends to infinity. In particular, $\lambda$ tends asymptotically to a non-random (" deterministic") quantity in agreement with general theory. 
In order to pass from the statistics of $\lambda$ to the statistics of the PS-field we introduce the fluctuating quantity

$$
Q\left(\frac{r}{L}\right) \equiv P_{2} \int_{0}^{\infty} d t J_{0}\left(\frac{r}{L} e^{\lambda t}\right)
$$

whose distribution can be restored from the PDF of $\lambda$. Indeed, at $\frac{r}{L} \ll 1$ we can cut the integral in the rhs of the Eq. (41) at $t=\frac{\ln (r / L)}{\lambda}$ and estimate $Q$ with logarithmic accuracy as

$$
Q\left(\frac{r}{L}\right) \approx \frac{P_{2}}{\lambda\left(\frac{\ln (L / r)}{\lambda}\right)} \ln \left(\frac{L}{r}\right) .
$$

It gives us the possibility to extract PDF of $Q$ from the expression (40). We obtain

$$
\mathcal{P}(Q) \approx \frac{D}{P_{2} \sqrt{2 \pi \ln \left(\frac{L}{r}\right)}} \exp \left[-\frac{\left(Q-\frac{P_{2}}{D} \ln \left(\frac{L}{r}\right)\right)^{2}}{2 P_{2} Q} D\right] .
$$

Such a PDF has at $r \rightarrow 0$ asymptotically the form of the Gaussian distribution for the quantity $\frac{Q}{\ln \left(\frac{L}{r}\right)}$ approaching the $\delta$-functional form when $\frac{L}{r} \rightarrow \infty$.

\section{ACKNOWLEDGMENTS}

We are grateful to G. Falkovich and V. Lebedev for helpful advises and numerous discussions. I.K. is grateful to U. Smilansky for the hospitality extended to him during his stay in the Weizmann Institute where the main part of this work was done. Y.V.F acknowledges with thanks the financial support of the Sir Charles Clore Postdoctoral Fellowship. 


\section{REFERENCES}

[1] G.K. Batchelor, J.Fluid Mech. 5, 113 (1959)

[2] R. Kraichnan, Phys. Fluids 10, 1417 (1967); J.Fluid Mech. 47, 525 (1971), 67 ,155 $(1975)$

[3] G. Falkovich and V.Lebedev, Preprint WIS-93/39/Apr-PH, Phys.Rev. E (to be published)

[4] V. L'vov, Phys. Reports 207, 1 (1991)

[5] Due to the fact that one supposes to have an inviscid flow.

[6] It is possible to show that in the case of the white noise statistics the arbitrary assymetry (which is compatible with the isotropy of the velocity field) does not effect all physical averages at all (see also [3]).

[7] I. Kolokolov, Ann. of Phys., 202, 165 (1990); I. Kolokolov and E. Podivilov, JETP., 68,119 (1989)

[8] I. Kolokolov, JETP., 76, 1099 (1993)

[9] I. Kolokolov, Phys. Lett., 114A, 99 (1986)

[10] It should be noted that the convergence is provided by the term $-\dot{\psi}^{+} \psi^{-}$in the action where the discretization (24) is assumed.

[11] W. Feller, An Introduction to probability theory and its applications (John Wiley and Sons, New York 1957)

[12] E. Le Page, in Probability Measures on Groups, A. Dold and B. Eckmann, eds, p.258 (Springer-Verlag 1982)

[13] V.I. Mel'nikov, JETP Lett, 32, 225 (1980)

[14] Y.V. Fyodorov and A.D. Mirlin, JETP Lett, 58 , 615, (1993), 615 
[15] I.M. Lifshitz, S. Gradescul and L.A. Pastur, Introduction to the theory of Disorded Systems (Wiley, New-York 1988) 